\title{
SARS-CoV-2 Variants: A Synopsis of In Vitro Efficacy Data of Convalescent Plasma, Currently Marketed Vaccines, and Monoclonal Antibodies
}

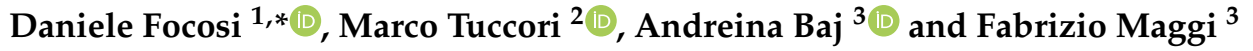 \\ 1 North-Western Tuscany Blood Bank, Pisa University Hospital, Via Paraisa 2, 56124 Pisa, Italy \\ 2 Division of Pharmacovigilance, Pisa University Hospital, Via Paradisa 2, 56124 Pisa, Italy; \\ m.tuccori@ao-pisa.toscana.it \\ 3 Department of Medicine and Surgery, University of Insubria, 21100 Varese, Italy; \\ andreina.baj@uninsubria.it (A.B.); fabrizio.maggi@uninsubria.it (F.M.) \\ * Correspondence: daniele.focosi@gmail.com
}

Citation: Focosi, D.; Tuccori, M.; Baj, A.; Maggi, F. SARS-CoV-2 Variants: A Synopsis of In Vitro Efficacy Data of Convalescent Plasma, Currently Marketed Vaccines, and Monoclonal Antibodies. Viruses 2021, 13, 1211. https://doi.org/10.3390/ v13071211

Received: 18 May 2021

Accepted: 2 June 2021

Published: 23 June 2021

Publisher's Note: MDPI stays neutral with regard to jurisdictional claims in published maps and institutional affiliations.

Copyright: (C) 2021 by the authors Licensee MDPI, Basel, Switzerland. This article is an open access article distributed under the terms and conditions of the Creative Commons Attribution (CC BY) license (https:/ / creativecommons.org/licenses/by/ $4.0 /)$.

\begin{abstract}
We summarize here in vitro evidences of efficacy for convalescent plasma, currently approved vaccines and monoclonal antibodies against SARS-CoV-2 variants of concern (VOC: B.1.1.7, B.1.351, P.1, and B.1.617.2), variants of interest (VOI: B.1.427/B.1.429, P.2, B.1.525, P.3, B.1.526, and B.1.671.1), and other strains (B.1.1.298 and B.1.258delta). While waiting from real world clinical efficacy, these data provide guidance for the treating physician.
\end{abstract}

Keywords: COVID-19; SARS-CoV-2; convalescent plasma; neutralizing antibody; immune escape; mutations; LyCoV016; etesevimab; REGN10987; imdevimab

The ongoing SARS-CoV-2 pandemic has entered a new dimension thanks to availability of different vaccines and neutralizing antibody-based therapeutics (from convalescent plasma to monoclonal antibodies). Nevertheless, emerging of SARS-CoV-2 variants of concern (VOC) and variants of interest (VOI) [1] has diversified the landscape, jeopardizing the efforts to contain it. Media-informed patients are questioning physicians about the relative efficacy of different vaccines and treatments against different variants.

The majority of registration trials for currently approved monoclonal antibodies and vaccines were run either before the variants emerged, or enrolled patients in countries where such variants were not circulating at that time. While waiting for post-marketing clinical efficacy data (i.e., protection from symptomatic COVID19), many investigators have tested the neutralizing efficacy of monoclonal antibodies, convalescent plasma from previous waves or vaccinee sera in vitro to accelerate availability of surrogate endpoints. Different nonviral (e.g., synthetic RBD) and viral constructs (ranging from isogenic strain, pseudovirus harboring the full mutation set, or authentic variant virus) have been employed to test the efficacy of therapeutics in neutralization assays [2].

On 15 May 2021, we mined PubMed and preprint servers (medrXiv and biorXiv) for in vitro data detailing the efficacy of different anti-Spike vaccines and monoclonal antibodies against different variants compared to wild-type SARS-CoV-2. Table 1 reports the result of our search and analysis. We decided to simplify interpretation of results using a semiquantitative scale according to the number of fold decrease in neutralization efficacy. We also tabulated for each variant the estimated reinfection rates, and the proven reinfection cases (strains from each episode sequenced). Each variant was reported using both the official (PANGOLIN and NextStrain) and the local (VUI/VOC/VOI) naming systems, and colloquial terms (e.g., "UK variant") in order to provide comprehensive association. The main, alarming finding is the lack of efficacy of single-agent bamlanivimab against most E484K-carrying variants. Accordingly, the FDA has recently withdrawn its emergency use authorization as a single agent, leaving the authorization only for usage 
in combination with etesevimab. Nevertheless, Q493R mutation, causing resistance to both $\mathrm{mAbs}$, has been recently reported by our group [3]. High-frequency Spike mutations R346K/S, N439K, G446V, L455F, V483F/A, E484Q/V/A/G/D, F486L, F490L/V/S, Q493R, and $\mathrm{S} 494 \mathrm{P} / \mathrm{L}$ might compromise some of mAbs in clinical trials [4].

We could not find any in vitro evidence of efficacy against SARS-CoV-2 variants for several widely used vaccines (e.g., Sinopharm's BBIBP-CorV), stressing the need for more studies.

Meta-analysis of 56 vaccine studies, including 2483 individuals and 8590 neutralization tests, showed that, compared with lineage B, there was a 1.5-fold reduction in neutralization against the B.1.1.7, 8.7-fold reduction against B.1.351 and 5.0-fold reduction against P.1. The estimated neutralization reductions for B.1.351 compared to lineage B were 240.2-fold reduction for non-replicating vector platform, 4.6-fold reduction for RNA platform, and 1.6-fold reduction for protein subunit platform. The neutralizing antibodies induced by administration of inactivated vaccines and mRNA vaccines against lineage P.1 were also remarkably reduced by an average of 5.9-fold and 1.5-fold [5]. Efficacy of convalescent plasma from previous waves is also generally lowered: convalescent plasma from a donor affected during the early 2020 protected against SARS-CoV-2 WA-1 wild-type strain but was insufficient to protect against challenge with B.1.1.7 and B.1.351 in a mouse model [6].

The relevance of these in vitro data in real life remains unclear. Khoury et al, assuming that the neutralization level required for $50 \%$ protection against detectable SARS-CoV-2 infection was $20.2 \%$ of the mean convalescent level and that the neutralization level for $50 \%$ protection from severe infection was 3\% of the mean convalescent level, reported that the decay of the neutralization titer over the first 250 days after immunization predicts a significant loss in protection from SARS-CoV-2 infection, although protection from severe disease should be largely retained. Neutralization titers against some SARS-CoV-2 VOC are reduced compared with the vaccine strain [7]. A case-control study showed that, compared with unvaccinated individuals, BNT162b2 vaccinees with documented SARSCoV-2 infection at least a week after the second dose were disproportionally infected with B.1.351 (odds ratio of 8:1), while those infected between 2 weeks after the first dose and 1 week after the second dose, were disproportionally infected by B.1.1.7 (odds ratio of 26:10), suggesting reduced vaccine efficacy (VE) against both VOCs under different dosage/timing conditions. Nevertheless, the B.1.351 incidence in Israel to-date remains low and VE remains high against B.1.1.7, among those fully vaccinated. These results overall suggest that vaccine breakthrough infection is more frequent with both VOCs, yet a combination of mass-vaccination with two doses coupled with non-pharmaceutical interventions control and contain their spread [8]. With mRNA-1273, binding of vaccineelicited anti-RBD antibodies is more broadly distributed across epitopes than for infectionelicited anti-RBD antibodies [9]: accordingly, greater IgG2, IgG3, and IgG4 responses and higher ratios of (IgG1 + IgG3)/(IgG2 + IgG4) were seen in subjects vaccinated with either BNT162b2 or mRNA-1273 than in convalescents [10]. This greater binding breadth means single RBD mutations have less impact on neutralization by vaccine sera than convalescent sera. Another striking feature is that for BNT162b2 post-first dose vaccination infection did not increase IgG titres, so that individuals infected post-dose one should receive the second [11]. CoronaVac for which no in vitro efficacy data are available, was $42 \%$ effective in the real-world setting of extensive P.1 transmission, but significant protection was not observed until completion of the two-dose regimen [12]. A metaanalysis by Shapiro et al found that, on average, the VE against any disease with infection was $85 \%$ after a full course of vaccination. The VE against severe disease, hospitalization or death averages close to $100 \%$. The average VE against infection, regardless of symptoms, was $84 \%$. The average VE against [13] B.1.1.28 (P1) and B.1.351 were 86\% (95\% CI: 65-84\%), 61\% (95\% CI: 43-73\%) and 56\% (95\% CI: $29-73 \%)$, respectively [14].

Additionally, a single injection of mRNA-1273 or BNT162b2 has been shown enough to induce novel antibody specificities that protect against the B.1.351 VOC [15]: a similar phenomenon has been reported after 2 BNT162b2 doses against B.1.1.7 [16]. Neutralizing antibody titers increased in previously infected BNT162b2 vaccinees relative to uninfected 
vaccinees against every variant tested: 5.2-fold against B.1.1.7, 6.5-fold against B.1.351, 4.3-fold against P.1, and 3.4-fold against original SARS-CoV-2 [17]. Similarly, a single dose of either BNT162b2 or AZD1222 vaccines in convalescents raised the titre of antibodies against the SARS-CoV-2 vaccine strain (B.1) and three major VOCs (B.1.1.7, B.1351 and P.1). A single dose to convalescents is nowadays a well-accepted approach that saves money and side effects [18].

Apart from efficacy, many topics remain under investigation for vaccines:

- $\quad$ vaccine-elicited T-cell immunity: while neutralizing antibodies are just one arm of the adaptive immune response to vaccines, very few data are available for protection from T-cell immunity, which would be especially relevant in the ones who do not mount antibody responses. Gallagher et al found detectable but diminished T-cell responses to Spike variants (B.1.1.7, B.1.351, and B.1.1.248) among BNT162b2 or mRNA-1273 vaccinated donors [19]. BNT162b2 or mRNA-1273-elicited spike-specific $\mathrm{T}$ cells responded similarly to stimulation by Spike epitopes from the ancestral, B.1.1.7 and B.1.351 variant strains, both in terms of cell numbers and phenotypes. In infectionnaive individuals, the second dose boosted the quantity but not quality of the $\mathrm{T}$ cell response, while in convalescents the second dose helped neither. Spike-specific $T$ cells from convalescent vaccinees differed strikingly from those of infection-naive vaccinees, with phenotypic features suggesting superior long-term persistence and ability to home to the respiratory tract including the nasopharynx [20].

- duration of protection: according to a mathematical model by Luo et al, after mRNA1273 vaccination, pseudovirus neutralization test against B.1.351 is expected to fall below the lower limit of detection of 20 geometric mean titers on day 100; variant P.1 on day 202, variant B.1.429 on day 258; and variant B.1.1.7 on day 309 [21]. Real-world data instead suggested that binding and functional antibodies against B.1.1.7, B.1.351, P.1, B.1.429, and B.1.526 variants persisted in most subjects, albeit at low levels, for 6 months after the primary series of mRNA-1273 [22].

- postponing second doses has been widely implemented in order to optimize vaccine delivery under manufacturing bottlenecks. In nonconvalescent elderlies higher than age 80 who received the second dose of BNT162b2 after 12 weeks instead of 3, the peak antibody response was 3.5-fold higher, but cellular immune responses were 3.6-fold lower [23].

- heterologous boosting: heterologous immunization strategy combining inactivated and mRNA vaccines can generate robust vaccine responses and therefore provide a rational and effective vaccination regimen [24]. ChAdOx/BNT162b2 booster vaccination was largely comparable to homologous BNT162b2/BNT162b2 vaccination and overall well-tolerated. No major differences were observed in the frequency or severity of local reactions after either of the vaccinations. In contrast, notable differences between the regimens were observed for systemic reactions, which were most frequent after prime immunization with ChAdOx (86\%) and less frequent after homologous BNT162b2/BNT162b2 (65\%), or heterologous ChAdOx/BNT162b2 boosters (48\%) [25]. Neutralizing activity against the prevalent strain B.1.1.7 was 3.9-fold higher than in individuals receiving homologous BNT162b2 vaccination, only 2-fold reduced for variant of concern B.1.351, and similar for variant B.1.617 [26]. Whilst both ChAdOx and BNT162b2 boosted prime-induced immunity, BNT induced significantly higher frequencies of Spike-specific CD4 and CD8 T cells and, in particular, high titers of neutralizing antibodies against the B.1.1.7, B.1.351 and the P.1 VOCs [27]. 
Table 1. Synopsis of efficacy of vaccines and monoclonal antibodies against current SARS-CoV-2 VOCs, VOIs and emerging strains.

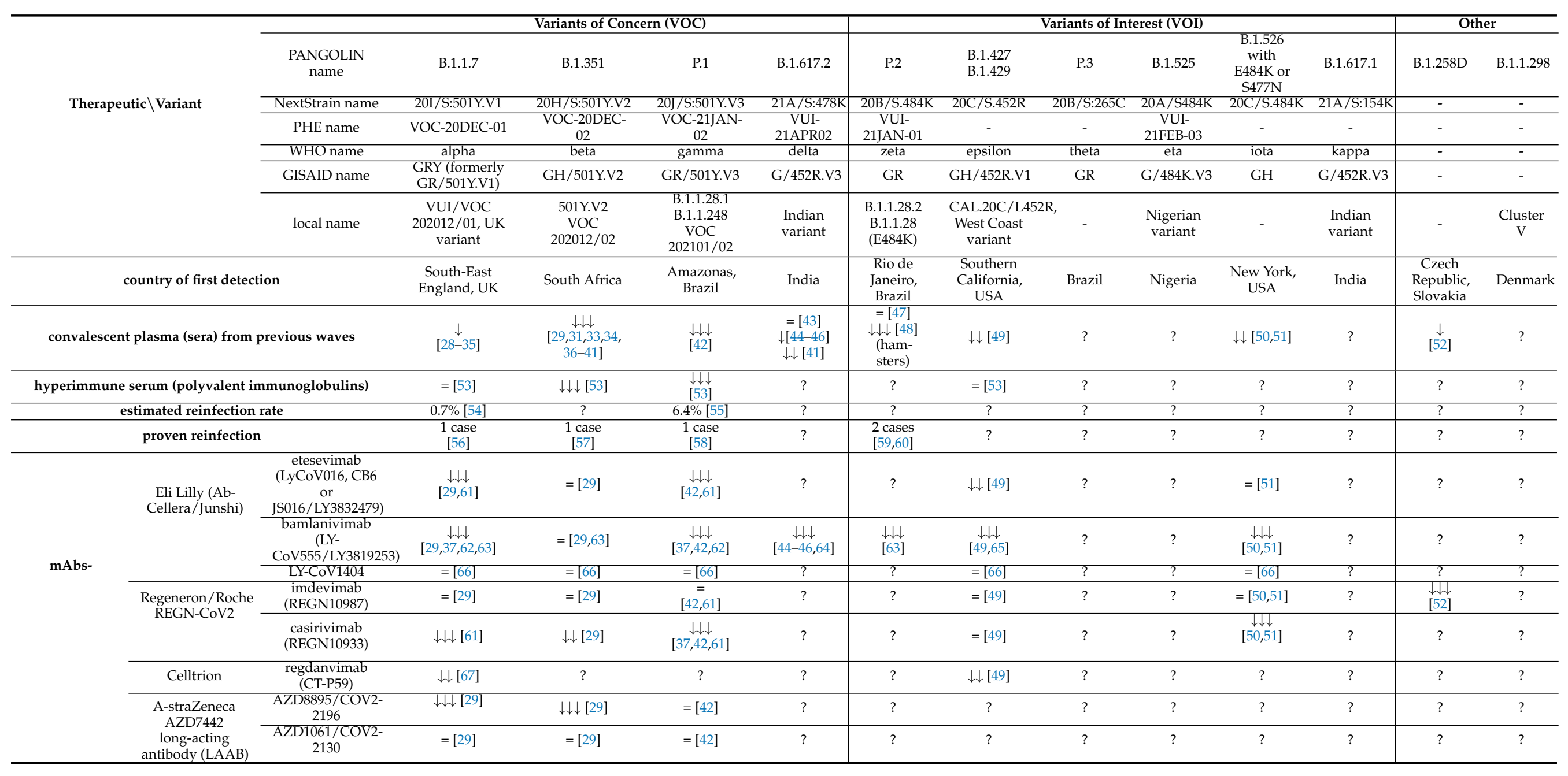


Table 1. Cont

\begin{tabular}{|c|c|c|c|c|c|c|c|c|c|c|c|c|c|c|}
\hline & \multicolumn{6}{|c|}{ Variants of Concern (VOC) } & \multicolumn{6}{|c|}{ Variants of Interest (VOI) } & \multicolumn{2}{|c|}{ Other } \\
\hline & BMS & C135 & $=[29]$ & $=[29]$ & $?$ & $?$ & $?$ & $?$ & $?$ & $?$ & $?$ & $?$ & $?$ & $?$ \\
\hline & DAVIS & C144 & $?$ & $?$ & $?$ & $?$ & $?$ & $?$ & $?$ & $?$ & $?$ & $?$ & ? & $?$ \\
\hline & $\begin{array}{c}\text { Vir } \\
\text { Biotechnology }\end{array}$ & $\begin{array}{c}\text { VIR-7831/GSK- } \\
4182136 \\
\text { (sotrovimab) and } \\
\text { VIR-7832/GSK- } \\
4182137 \text { (both } \\
\text { derived from } \\
\text { S309) } \\
\end{array}$ & $=[29,68]$ & $=[29,68]$ & $=[42,68]$ & ? & $?$ & $?$ & ? & $?$ & ? & $?$ & ? & ? \\
\hline \multirow[t]{7}{*}{ vaccine } & BioNtech/Pfizer & $\begin{array}{l}\text { BNT162b2/ } \\
\text { tozinameran } \\
\left.\text { (Comirnaty }{ }^{\circledR}\right)\end{array}$ & $\begin{array}{c}=[69] \\
\downarrow \\
{[15,29,31,33,35,37,63,} \\
70-75] \\
\text { RBD } 1 \downarrow \downarrow[76-78] \\
\text { COVID19 } 75 \%[79]\end{array}$ & $\begin{array}{c}=/ \downarrow \\
{[29,31-} \\
33,35,63,70- \\
75,80-82] \\
\text { RBD [78] } \\
\text { COVID19 90\% } \\
\text { [79,83]-93\% } \\
\text { [84] }\end{array}$ & $\underset{[35,42,75,76,85]}{\downarrow}$ & $\begin{array}{c}\downarrow \downarrow \downarrow[44- \\
46,86,87] \\
\text { COVID19 } \\
88 \% \text { [84] }\end{array}$ & {$\left[\begin{array}{c}\downarrow \\
{[63]}\end{array}\right]$} & $\begin{array}{c}=/ \downarrow \downarrow[49,75] \\
\text { RBD }[78]\end{array}$ & ? & $\downarrow[88]$ & $\downarrow \downarrow[50,51]$ & $?$ & $?$ & $=[75]$ \\
\hline & Moderna & mRNA-1273 & $\begin{array}{c}\downarrow 15,29,38,74,75,89] \\
\downarrow \text { NHP COVIID19 } \\
{[90]}\end{array}$ & $\begin{array}{c}= \\
{[29,30,89]} \\
\downarrow[74,75,91,92]\end{array}$ & $=/ \downarrow[42,75]$ & $\downarrow \downarrow[44,87]$ & ? & $=/ \downarrow \downarrow[49,75]$ & ? & ? & $\downarrow \downarrow[50,51]$ & $?$ & ? & $=[75]$ \\
\hline & AstraZeneca & $\begin{array}{l}\text { AZD1222/ChAdOx1 } \\
\text { (Vaxzevria® } \\
\left.\text { Covishield }{ }^{\circledR}\right)^{\prime}\end{array}$ & $\begin{array}{c}\downarrow \downarrow \downarrow \downarrow \\
{[93]} \\
=\text { hamster COVID19 } \\
{[94]} \\
\text { COVID19 22\% } \\
\end{array}$ & $\begin{array}{c}? \\
\text { COVID19 90\% } \\
\text { [83]-66\% [84] } \\
=\text { hamster } \\
\text { COVID19 [94] }\end{array}$ & $\downarrow \downarrow[85]$ & $\begin{array}{c}\downarrow[45,95] \\
\text { COVID19 } \\
\%[84]\end{array}$ & ? & $?$ & ? & $?$ & $?$ & $?$ & $?$ & ? \\
\hline & Novovax & $\begin{array}{c}\text { NVX-CoV2373 } \\
\left(\text { Covovax }^{\oplus}\right)\end{array}$ & COVID19: 51\% [97] & $\downarrow[92]$ & ? & $?$ & ? & ? & ? & ? & $?$ & $?$ & ? & $?$ \\
\hline & Bharat Biotech & BBV152/Covaxin & $?$ & $\begin{array}{l}=[98] \\
\downarrow[41]\end{array}$ & $?$ & $\begin{array}{l}=[43] \\
\downarrow[41]\end{array}$ & $\downarrow[47]$ & $?$ & $?$ & $?$ & $?$ & $?$ & $?$ & $?$ \\
\hline & SinoVac & CoronaVac & $\downarrow \downarrow \downarrow[99]$ & $=[99]$ & $\downarrow \downarrow[99]$ & $?$ & $?$ & $=[99]$ & $?$ & $?$ & $\downarrow \downarrow[99]$ & $?$ & $?$ & $?$ \\
\hline & J\&J/Janssen & $\begin{array}{l}\text { JNJ- } 26 . C O V \\
\text { 78436735/Ad26.COV }\end{array}$ & 2.5 & $\downarrow \downarrow[100]$ & $?$ & $?$ & $?$ & $?$ & $?$ & $?$ & $?$ & $?$ & $?$ & $?$ \\
\hline
\end{tabular}

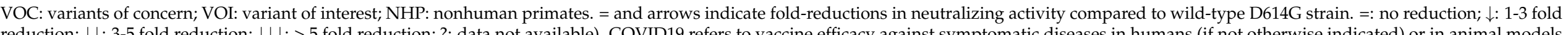

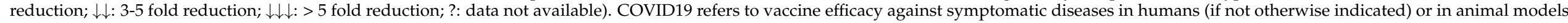
(specified). RBD: ACE2-RBD competition assay. 
While the in vitro findings summarized here wait for confirmatory clinical evidences, in the meanwhile they could orient therapeutic and preventive strategies.

Author Contributions: D.F. conceived the design and wrote the first draft. F.M., M.T. and A.B. critically revised the final version. All authors have read and agreed to the published version of the manuscript.

Funding: This research received no external funding.

Data Availability Statement: The data presented in this study are openly available in PubMed.

Conflicts of Interest: We declare we have no conflict of interest related to this manuscript.

\author{
Abbreviations \\ $\mathrm{nAb}$ neutralizing antibodies \\ CCP COVID19 convalescent plasma \\ RBD receptor-binding domain \\ RBM receptor-binding motif
}

\title{
References
}

1. WHO. COVID-19 Weekly Epidemiological Update 25 February 2021. Special Edition: Proposed Working Definitions of SARSCoV-2 Variants of Interest and Variants of Concern. 2021. Available online: https://www.who.int/publications/m/item/covid19-weekly-epidemiological-update (accessed on 17 June 2021).

2. Focosi, D.; Mazzetti, P.; Pistello, M.M. Viral infection neutralization tests: A focus on SARS-CoV-2 with implications for convalescent plasma therapy. Rev. Med. Virol. 2020, 31, e2170. [PubMed]

3. Focosi, D.; Novazzi, F.; Genoni, A.; Dentali, F.; Dalla Gasperina, D.; Baj, A.; Maggi, F. Emergence of SARS-CoV-2 Spike Escape Mutation Q493R After Bamlanivimab/Etesevimab Treatment for COVID-19. Res. Sq. 2021. [CrossRef]

4. Chen, J.; Gao, K.; Wang, R.; Wei, G.-W. Revealing the threat of emerging SARS-CoV-2 mutations to antibody therapies. bioRxiv 2021. [CrossRef]

5. Chen, X.; Chen, Z.; Azman, A.S.; Sun, R.; Lu, W.; Zheng, N.; Zhou, J.; Wu, Q.; Deng, X.; Zhao, Z.; et al. Comprehensive mapping of neutralizing antibodies against SARS-CoV-2 variants induced by natural infection or vaccination. medRxiv 2021. [CrossRef]

6. Horspool, A.M.; Ye, C.; Wong, T.Y.; Russ, B.P.; Lee, K.S.; Winters, M.T.; Bevere, J.R.; Kieffer, T.; Martinez, I.; Sourimant, J.; et al. SARS-CoV-2 B.1.1.7 and B.1.351 variants of concern induce lethal disease in K18-hACE2 transgenic mice despite convalescent plasma therapy. bioRxiv 2021. [CrossRef]

7. Khoury, D.S.; Cromer, D.; Reynaldi, A.; Schlub, T.E.; Wheatley, A.K.; Juno, J.A.; Subbarao, K.; Kent, S.J.; Triccas, J.A.; Davenport, M.P. Neutralizing antibody levels are highly predictive of immune protection from symptomatic SARS-CoV-2 infection. Nat. Med. 2021, 1-7. [CrossRef]

8. Kustin, T.; Harel, N.; Finkel, U.; Perchik, S.; Harari, S.; Tahor, M.; Caspi, I.; Levy, R.; Leshchinsky, M.; Ken Dror, S.; et al. Evidence for increased breakthrough rates of SARS-CoV-2 variants of concern in BNT162b2 mRNA vaccinated individuals. Nat. Med. 2021. [CrossRef]

9. Greaney, A.J.; Loes, A.N.; Gentles, L.E.; Crawford, K.H.D.; Starr, T.N.; Malone, K.D.; Chu, H.Y.; Bloom, J.D. The SARS-CoV-2 mRNA-1273 vaccine elicits more RBD-focused neutralization, but with broader antibody binding within the RBD. bioRxiv 2021. [CrossRef]

10. Klingler, J.; Gregory, S.; Itri, V.; Liu, S.; Bandres, J.C.; Enyindah-Asonye, G.; Liu, X.; Oguntuyo, K.Y.; Amanat, F.; Lee, B. SARSCoV-2 mRNA vaccines induce a greater array of spike-specific antibody isotypes with more potent complement binding capacity than natural infection. medRxiv 2021. [CrossRef]

11. Abu Jabal, K.; Ben Amram, H.; Beiruti, K.; Brimat, I.; Abu Saaa, A.; Batish, Y.; Sussan, C.; Zarka, S.; Edelstein, M. SARS-CoV-2 Immunogenicity in individuals infected before and after COVID-19 vaccination: Israel, January-March 2021: Implications for vaccination policy. medRxiv 2021. [CrossRef]

12. Ranzani, O.T.; Hitchings, M.; Dorion Neto, M.; Lang D’Agostini, T.; Cardoso de Paulha, R.; Ferreira Pereira de Paula, O.; de Moura Villela, F.; Scaramuzzini Torres, M.S.; Barbosa de Oliveira, M.; Schulz, W.; et al. Effectiveness of the CoronaVac vaccine in the elderly population during a P.1 variant-associated epidemic of COVID-19 in Brazil: A test-negative case-control study. medRxiv 2021. [CrossRef]

13. Nadesalingam, A.; Cantoni, D.; Wells, D.A.; Aguinam, E.T.; Ferrari, M.; Smith, P.; Chan, A.; Carnell, G.; Ohlendorf, L.; Einhauser, S.; et al. Breadth of neutralising antibody responses to SARS-CoV-2 variants of concern is augmented by vaccination following prior infection: Studies in UK healthcare workers and immunodeficient patients. medrXiv 2021. [CrossRef]

14. Shapiro, J.; Dean, N.E.; Madewell, Z.J.; Yang, Y.; Halloran, M.E.; Longini, I.M. Efficacy Estimates for Various COVID-19 Vaccines: What we Know from the Literature and Reports. medRxiv 2021. [CrossRef] 
15. Stamatatos, L.; Czartoski, J.; Wan, Y.-H.; Homad, L.J.; Rubin, V.; Glantz, H.; Nerdilek, M.; Seydoux, E.; Jennewein, M.F.; MacCamy, A.J.; et al. mRNA vaccination boosts cross-variant neutralizing antibodies elicited by SARS-CoV-2 infection. Science 2021, eabg9175. [CrossRef]

16. Trinite, B.; Pradenas, E.; Marfil, S.; Rovirosa, C.; Urrea, V.; Tarres-Freixas, F.; Ortiz, R.; Rodon, J.; Veragara-Alert, J.; Segales, J.; et al. Previous SARS-CoV-2 infection increases B.1.1.7 cross-neutralization by vaccinated individuals. Viruses 2021, 13, 1135. [CrossRef]

17. Leier, H.C.; Bates, T.A.; Lyski, Z.L.; McBride, S.K.; Lee, D.X.; Coulter, F.J.; Goodman, J.R.; Lu, Z.; Curlin, M.E.; Messer, W.B.; et al. Previously infected vaccinees broadly neutralize SARS-CoV-2 variants. medRxiv 2021. [CrossRef]

18. Focosi, D.; Baj, A.; Maggi, F. Is a single COVID-19 vaccine dose enough in convalescents? Hum. Vaccines Immunother. 2021, 1-3. [CrossRef]

19. Gallagher, K.M.E.; Leick, M.B.; Larson, R.C.; Berger, T.R.; Katsis, K.; Yam, J.Y.; Brini, G.; Grauwet, K. MGH COVID-19 Collection \& Processing Team; Maus, M.V. SARS -CoV-2 T-cell immunity to variants of concern following vaccination. bioRxiv 2021. [CrossRef]

20. Neidleman, J.; Luo, X.; McGregor, M.; Xie, G.; Murray, V.; Greene, W.C.; Lee, S.A.; Roan, N.R. mRNA vaccine-induced SARSCoV-2-specific T cells recognize B.1.1.7 and B.1.351 variants but differ in longevity and homing properties depending on prior infection status. bioRxiv 2021. [CrossRef]

21. Luo, G.; Hu, Z.; Letterio, J. Modeling and Predicting Antibody Durability for mRNA-1273 Vaccine for SARS-CoV-2 Variants. medRxiv 2021. [CrossRef]

22. Pegu, A.; O'Connell, S.E.; Schmidt, S.D.; O’Dell, S.; Talana, C.A.; Lai, L.; Albert, J.; Anderson, E.; Bennett, H.; Corbett, K.; et al. Durability of mRNA-1273-induced antibodies against SARS-CoV-2 variants. bioRxiv 2021. [CrossRef]

23. Parry, H.M.; Bruton, R.; Stephens, C.; Brown, K.; Amirthalingam, G.; Hallis, B.; Otter, A.; Zuo, J.; Moss, P. Extended interval BNT162b2 vaccination enhances peak antibody generation in older people. medRxiv 2021. [CrossRef]

24. Lin, A.; Liu, J.; Ma, X.; Zhao, F.; Yu, B.; He, J.; Shen, M.; Huang, L.; Tang, H.; Jiang, E.; et al. Heterologous vaccination strategy for containing COVID-19 pandemic. medRxiv 2021. [CrossRef]

25. Hillus, D.; Tober-Lau, P.; Hastor, H.; Helbig, E.T.; Lippert, L.J.; Thibeault, C.; Solarek, A.; Kalle, C.V.; Corman, V.M.; Kopankiewicz, P.; et al. Reactogenicity of homologous and heterologous prime-boost immunization with BNT162b2 and ChAdOx1-nCoV19: A prospective cohort study. medRxiv 2021. [CrossRef]

26. Gross, R.; Zanoni, M.; Seidel, A.; Conzelmann, C.; Gilg, A.; Krnavek, D.; Erdemci-evin, S.; Mayer, B.; Hoffmann, M.; Poehlmann, S.; et al. Heterologous ChAdOx1 nCoV-19 and BNT162b2 prime-boost vaccination elicits potent neutralizing antibody responses and $\mathrm{T}$ cell reactivity. medrXiv 2021. [CrossRef]

27. Barros-Martins, J.; Hammerschmidt, S.; Cossmann, A.; Odak, I.; Stankov, M.V.; Morillas Ramos, G.; Jablonka, A.; Heidemann, A.; Ritter, C.; Friedrichsen, M.; et al. Humoral and cellular immune response against SARS-CoV-2 variants following heterologous and homologous ChAdOx1 nCoV-19/BNT162b2 vaccination. medrXiv 2021. [CrossRef]

28. Rees-Spear, C.; Muir, L.; Griffith, S.A.; Heaney, J.; Aldon, Y.; Snitselaar, J.; Thomas, P.; Graham, C.; Seow, J.; Lee, N.; et al. The impact of Spike mutations on SARS-CoV-2 neutralization. Cell Rep. 2021, 34, 108890. [CrossRef] [PubMed]

29. Wang, P.; Nair, M.S.; Lihong, L.; Iketani, S.; Luo, Y.; Guo, Y.; Wang, M.; Yu, J.; Zhang, B.; Kwong, P.D.; et al. Antibody resistance of SARS-CoV-2 variants B.1.351 and B.1.1.7. Nature 2021, 593, 130-135. [CrossRef] [PubMed]

30. Edara, V.V.; Floyd, K.; Lai, L.; Gardner, M.; Hudson, W.; Piantadosi, A.; Waggoner, J.; Babiker, A.; Ahmed, R.; Xie, X.; et al. Infection and mRNA-1273 vaccine antibodies neutralize SARS-CoV-2 UK variant. medRxiv 2021. [CrossRef]

31. Planas, D.; Bruel, T.; Grzelak, L.; Guivel-Benhassine, F.; Staropoli, I.; Porrot, F.; Planchais, C.; Buchrieser, J.; Rajah, M.M.; Bishop, E.; et al. Sensitivity of infectious SARS-CoV-2 B.1.1.7 and B.1.351 variants to neutralizing antibodies. Nat. Med. 2021, 27, 917-924. [CrossRef] [PubMed]

32. Brown, J.C.; Goldhill, D.H.; Zhou, J.; Peacock, T.P.; Frise, R.; Goonawardane, N.; Baillon, L.; Kugathasan, R.; Pinto, A.; McKay, P.F.; et al. Increased transmission of SARS-CoV-2 lineage B.1.1.7 (VOC 2020212/01) is not accounted for by a replicative advantage in primary airway cells or antibody escape. BioRxiv 2021. [CrossRef]

33. Bates, T.A.; Leier, H.C.; Lyski, Z.L.; McBride, S.K.; Coulter, F.J.; Weinstein, J.B.; Goodman, J.R.; Lu, Z.; Siegel, S.A.R.; Sullivan, P.; et al. Neutralization of SARS-CoV-2 variants by convalescent and vaccinated serum. medRxiv 2021. [CrossRef]

34. Fenwick, C.; Turelli, P.; Pellaton, C.; Farina, A.; Campos, J.; Raclot, C.; Pojer, F.; Cagno, V.; Pantaleo, G.; Trono, D. A multiplexed high-throughput neutralization assay reveals a lack of activity against multiple variants after SARS-CoV-2 infection. medRxiv 2021. [CrossRef]

35. Caniels, T.G.; Bontjer, I.; van der Straten, K.; Poniman, M.; Burger, J.A.; Appelman, B.; Lavell, A.H.A.; Oomen, M.; Godeke, G.-J.; Valle, C.; et al. Emerging SARS-CoV-2 variants of concern evade humoral immune responses from infection and vaccination. medrXiv 2021. [CrossRef]

36. Wibmer, C.K.; Ayres, F.; Hermanus, T.; Madzivhandila, M.; Kgagudi, P.; Lambson, B.E.; Vermeulen, M.; van den Berg, K.; Rossouw, T.; Boswell, M.; et al. SARS-CoV-2 501Y.V2 escapes neutralization by South African COVID-19 donor plasma. Nat. Med. 2021. [CrossRef]

37. Hoffmann, M.; Arora, P.; Gross, R.; Seidel, A.; Hoernich, B.; Hahn, A.; Krueger, N.; Graichen, L.; Hofmann-Winkler, H.; Kempf, A.; et al. SARS-CoV-2 variants B.1.351 and B.1.1.248: Escape from therapeutic antibodies and antibodies induced by infection and vaccination. medRxiv 2021. [CrossRef] 
38. Edara, V.V.; Norwood, C.; Floyd, K.; Lai, L.; Davis-Gardner, M.E.; Hudson, W.H.; Mantus, G.; Nyhoff, L.E.; Adelman, M.W.; Fineman, R.; et al. Reduced binding and neutralization of infection- and vaccine-induced antibodies to the B.1.351 (South African) SARS-CoV-2 variant. bioRxiv 2021. [CrossRef]

39. Cele, S.; Gazy, I.; Jackson, L.; Hwa, S.-H.; Tegally, H.; Lustig, G.; Giandhari, J.; Pillay, S.; Wilkinson, E.; Naidoo, Y.; et al. Escape of SARS-CoV-2 501Y.V2 variants from neutralization by convalescent plasma. Nature 2021, 593, 142-146. [CrossRef]

40. Riou, C.; Keeton, R.; Moyo-Gwete, T.; Hermanus, T.; Kgagudi, P.; Baguma, R.; Tegally, H.; Doolabh, D.; Iranzadeh, A.; Tyers, L.; et al. Loss of recognition of SARS-CoV-2 B.1.351 variant spike epitopes but overall preservation of T cell immunity. medrXiv 2021. [CrossRef]

41. Yadav, P.D.; Sapkal, G.; Ella, R.; Sahay, R.R.; Nyayanit, D.A.; Patil, D.Y.; Deshpande, G.; Shete, A.M.; Gupta, N.; Mohan, V.K.; et al. Neutralization against B.1.351 and B.1.617.2 with sera of COVID-19 recovered cases and vaccinees of BBV152. medrXiv 2021. [CrossRef]

42. Wang, P.; Wang, M.; Yu, J.; Cerutti, G.; Nair, M.S.; Huang, Y.; Kwong, P.D.; Shapiro, L.; Ho, D.D. Increased Resistance of SARS-CoV-2 Variant P.1 to Antibody Neutralization. Cell Host Microbe 2021, 29, 747-751. [CrossRef] [PubMed]

43. Yadav, P.; Sapkal, G.N.; Abraham, P.; Ella, R.; Deshpande, G.; Patil, D.Y.; Nyayanit, D.; Gupta, N.; Sahay, R.R.; Shete, A.M.; et al Neutralization of variant under investigation B.1.617 with sera of BBV152 vaccinees. Clin. Infect. Dis. 2021, ciab411. [CrossRef] [PubMed]

44. Tada, T.; Zhou, H.; Dcosta, B.M.; Samanovic, M.I.; Mulligan, M.J.; Landau, N.R. The Spike Proteins of SARS-CoV-2 B.1.617 and B.1.618 Variants Identified in India Provide Partial Resistance to Vaccine-elicited and Therapeutic Monoclonal Antibodies. medRxiv 2021. [CrossRef]

45. Planas, D.; Veyer, D.; Baidaliuk, A.; Staropoli, I.; Guivel-Benhassine, F.; Rajah, M.; Planchais, C.; Porrot, F.; Robillard, N.; Puech, J.; et al. Reduced sensitivity of infectious SARS-CoV-2 variant B.1.617.2 to monoclonal antibodies and sera from convalescent and vaccinated individuals. medRxiv 2021. [CrossRef]

46. Hoffmann, M.; Hofmann-Winkler, H.; Krueger, N.; Kempf, A.; Nehlmeier, I.; Graichen, L.; Sidarovich, A.; Moldenhauer, A.-S.; Winkler, M.S.; Schulz, S.; et al. SARS-CoV-2 variant B.1.617 is resistant to Bamlanivimab and evades antibodies induced by infection and vaccination. medRxiv 2021. [CrossRef]

47. Sapkal, G.; Yadav, P.D.; Ella, R.; Abraham, P.; Patil, D.Y.; Gupta, N.; Panda, S.; Mohan, V.K.; Bhargava, B. Neutralization of B.1.1.28 $\mathrm{P} 2$ variant with sera of natural SARS-CoV-2 infection and recipients of inactivated COVID-19 vaccine Covaxin. J. Travel Med. 2021, taab077. [CrossRef]

48. Yadav, P.; Mohandas, S.; Sarkale, P.; Nyayanit, D.; Shete, A.; Sahay, R.; Potdar, V.; Baradkar, S.; Gupta, N.; Sapkal, G.; et al. Isolation of SARS-CoV-2 B.1.1.28.2 P2 variant and pathogenicity comparison with D614G variant in hamster model. medrXiv 2021. [CrossRef]

49. McCallum, M.; Bassi, J.; De Marco, A.; Chen, A.; Walls, A.C.; Di Iulio, J.; Tortorici, M.A.; Navarro, M.-J.; Silacci-Fregni, C.; Saliba, C.; et al. SARS-CoV-2 immune evasion by variant B.1.427/B.1.429. biorXiv 2021. [CrossRef]

50. Zhou, H.; Dcosta, B.M.; Samanovic, M.I.; Mulligan, M.J.; Landau, N.R.; Tada, T.B. 1.526 SARS-CoV-2 variants identified in New York City are neutralized by vaccine-elicited and therapeutic monoclonal antibodies. biorXiv 2021. [CrossRef]

51. Annavajhala, M.K.; Mohri, H.; Zucker, J.E.; Sheng, Z.; Wang, P.; Gomez-Simmonds, A.; Ho, D.D.; Uhlemann, A.-C. A Novel SARS-CoV-2 Variant of Concern, B.1.526, Identified in New York. medrXiv 2021. [CrossRef]

52. Thomson, E.C.; Rosen, L.E.; Shepherd, J.G.; Spreafico, R.; da Silva Filipe, A.; Wojcechowskyj, J.A.; Davis, C.; Piccoli, L.; Pascall, D.J.; Dillen, J.; et al. The circulating SARS-CoV-2 spike variant N439K maintains fitness while evading antibody-mediated immunity. Cell 2021, 184, 1171-1187. [CrossRef]

53. Tang, J.; Lee, Y.; Ravichandran, S.; Grubbs, G.; Huang, C.; Stauft, C.; Wang, T.; Golding, B.; Golding, H.; Khurana, S. Reduced neutralization of SARS-CoV-2 variants by convalescent plasma and hyperimmune intravenous immunoglobulins for treatment of COVID-19. medrXiv 2021. [CrossRef]

54. Graham, M.S.; Sudre, C.H.; May, A.; Antonelli, M.; Murray, B.; Varsavsky, T.; Klaser, K.; Canas, L.D.S.; Molteni, E.; Modat, M.; et al. Changes in symptomatology, reinfection, and transmissibility associated with the SARS-CoV-2 variant B.1.1.7: An ecological study. Lancet Public Health 2021, 6, e335-e345. [CrossRef]

55. Coutinho, R.M.; Marquitti, F.M.D.; Ferreira, L.S.; Borges, M.E.; Silva, R.L.P.d.; Canton, O.; Portella, T.P.; Lyra, S.P.; Franco, C.; Silva, A.D.; et al. Model-based evaluation of transmissibility and reinfection for the P.1 variant of the SARS-CoV-2. medrXiv 2021. [CrossRef]

56. Harrington, D.; Kele, B.; Pereira, S.; Couto-Parada, X.; Riddell, A.; Forbes, S.; Dobbie, H.; Cutino-Moguel, T. Confirmed Reinfection with SARS-CoV-2 Variant VOC-202012/01. Clin. Infect. Dis. 2021, ciab0. [CrossRef]

57. Zucman, N.; Uhel, F.; Descamps, D.; Roux, D.; Ricard, J.D. Severe reinfection with South African SARS-CoV-2 variant 501Y.V2: A case report. Clin. Infect. Dis. 2021, ciab129. [CrossRef]

58. Naveca, F.; da Costa, C.; Nascimento, V.; Souza, V.; Corado, A.; Nascimento, F.; Costa, A.; Duarte, D.; Silva, G.; Mejía, M.; et al. SARS-CoV-2 Reinfection by the New Variant of Concern (VOC) P.1 in Amazonas, Brazil. Available online: https://virological. org/t/sars-cov-2-reinfection-by-the-new-variant-of-concern-voc-p-1-in-amazonas-brazil/596 (accessed on 27 January 2021).

59. Resende, P.; Bezerra, J.; de Vasconcelos, R.; Arantes, I.; Appolinario, L.; Mendonça, A.; Paixao, A.; Duarte Rodrigues, A.; Silva, T.; Rocha, A.; et al. Spike E484K mutation in the first SARS-CoV-2 reinfection case confirmed in Brazil. 2020. Available online: 
https:/ / virological.org/t/spike-e484k-mutation-in-the-first-sars-cov-2-reinfection-case-confirmed-in-brazil-2020/584 (accessed on 17 June 2021).

60. Nonaka, C.; Miranda Franco, M.; Gräf, T.; Mendes, A.V.A.; de Aguiar, R.S.; Giovanetti, M.; Solano de Freitas Souza, B. Genomic Evidence of a Sars-Cov-2 Reinfection Case with E484K Spike Mutation in Brazil. Emerg. Infect. Dis. 2021, 27. [CrossRef]

61. Wang, R.; Zhang, Q.; Ge, J.; Ren, W.; Zhang, R.; Lan, J.; Ju, B.; Su, B.; Yu, F.; Chen, P.; et al. Spike mutations in SARS-CoV-2 variants confer resistance to antibody neutralization. biorXiv 2021. [CrossRef]

62. Liu, H.; Wei, P.; Zhang, Q.; Chen, Z.; Aviszus, K.; Downing, W.; Peterson, S.; Reynoso, L.; Downey, G.; Frankel, S.; et al. 501Y.V2 and 501Y.V3 variants of SARS-CoV-2 lose binding to Bamlanivimab in vitro. MAbs 2021, 13, 1919285. [CrossRef]

63. Widera, M.; Wilhelm, A.; Hoehl, S.; Pallas, C.; Kohmer, N.; Wolf, T.; Rabenau, H.F.; Corman, V.M.; Drosten, C.; Vehreschild, M.J. Bamlanivimab does not neutralize two SARS-CoV-2 variants carrying E484K in vitro. medrXiv 2021. [CrossRef]

64. Zhang, L.; Huynh, T.; Luan, B. In silico Assessment of Antibody Drug Resistance to Bamlanivimab of SARS-CoV-2 Variant B.1.617. medrXiv 2021. [CrossRef]

65. Starr, T.N.; Greaney, A.J.; Dingens, A.S.; Bloom, J.D. Complete map of SARS-CoV-2 RBD mutations that escape the monoclonal antibody LY-CoV555 and its cocktail with LY-CoV016. Cell Rep. Med. 2021, 2, 100255. [CrossRef] [PubMed]

66. Westendorf, K.; Žentelis, S.; Foster, D.; Vaillancourt, P.; Wiggin, M.; Lovett, E.; Hendle, J.; Pustilnik, A.; Sauder, J.M.; Kraft, L.; et al. LY-CoV1404 potently neutralizes SARS-CoV-2 variants. biorXiv 2021. [CrossRef]

67. Lee, S.-Y.; Ryu, D.-K.; Choi, Y.K.; Moore, P.; Baalen, C.A.V.; Song, R.; Tijsma, A.S.; Gwete-Moyo, T.; Kim, M.; Kim, Y.-I.; et al. Therapeutic effect of CT-P59 against SARS-CoV-2 South African variant. medrXiv 2021. [CrossRef]

68. Cathcart, A.L.; Havenar-Daughton, C.; Lempp, F.A.; Ma, D.; Schmid, M.; Agostini, M.L.; Guarino, B.; Di iulio, J.; Rosen, L.; Tucker, H.; et al. The dual function monoclonal antibodies VIR-7831 and VIR-7832 demonstrate potent in vitro and in vivo activity against SARS-CoV-2. medrXiv 2021. [CrossRef]

69. Becker, M.; Dulovic, A.; Junker, D.; Ruetalo, N.; Kaiser, P.; Pinilla, Y.; Heinzel, C.; Haering, J.; Traenkle, B.; Wagner, T.; et al. Immune response to SARS-CoV-2 variants of concern in vaccinated individuals. Nat. Commun. 2021, 12, 3109. [CrossRef]

70. Xie, X.; Liu, Y.; Liu, J.; Zhang, X.; Zou, J.; Fontes-Garfias, C.R.; Xia, H.; Swanson, K.A.; Cutler, M.; Cooper, D. Neutralization of spike 69/70 deletion, E484K, and N501Y SARS-CoV-2 by BNT162b2 vaccine-elicited sera. Nat. Med. 2021, 27, 620-621. [CrossRef]

71. Tada, T.; Dcosta, B.M.; Samanovic-Golden, M.; Herati, R.S.; Cornelius, A.; Mulligan, M.J.; Landau, N.R. Neutralization of viruses with European, South African, and United States SARS-CoV-2 variant spike proteins by convalescent sera and BNT162b2 mRNA vaccine-elicited antibodies. biorXiv 2021. [CrossRef]

72. Kuzmina, A.; Khalaila, Y.; Voloshin, O.; Keren-Naus, A.; Bohehm, L.; Raviv, Y.; Shemer-Avni, Y.; Rosenberg, E.; Taube, R. SARS $\mathrm{CoV}-2$ escape variants exhibit differential infectivity and neutralization sensitivity to convalescent or post-vaccination sera. Cell Host Microbe 2021, 29, 522-528. [CrossRef]

73. Gonzalez, C.; Saade, C.; Bal, A.; Valette, M.; Saker, K.; Lina, B.; Josset, L.; Trabaud, M.-A.; Thiery, G.; Botelho-Nevers, E.; et al. Live virus neutralization testing in convalescent and vaccinated subjects against 19A, 20B, 20I/501Y.V1 and 20H/501.V2 isolates of SARS-CoV-2. medrXiv 2021. [CrossRef]

74. Liu, J.; Bodnar, B.H.; Wang, X.; Wang, P.; Meng, F.; Khan, A.I.; Saribas, A.S.; Padhiar, N.H.; McCluskey, E.; Shah, S.; et al. Correlation of vaccine-elicited antibody levels and neutralizing activities against SARS-CoV-2 and its variants. biorXiv 2021. [CrossRef]

75. Garcia-Beltran, W.F.; Lam, E.C.; Denis, K.S.; Nitido, A.D.; Garcia, Z.H.; Hauser, B.M.; Feldman, J.; Pavlovic, M.N.; Gregory, D.J.; Poznansky, M.C.; et al. Multiple SARS-CoV-2 variants escape neutralization by vaccine-induced humoral immunity. Cell 2021, 184, 2372-2383. [CrossRef]

76. Mohsen, M.; Bachmann, M.F.; Vogel, M.; Augusto, G.S.; Liu, X.; Chang, X. BNT162b2 mRNA COVID-19 vaccine induces antibodies of broader cross-reactivity than natural infection but recognition of mutant viruses is up to 10-fold reduced. Allergy 2021. [CrossRef]

77. Stankov, M.; Cossmann, A.; Bonifacius, A.; Jablonka, A.; Morillas Ramos, G.; Goedecke, N.; Zychlinsky Scharff, A.; Happle, C.; Boeck, A.-L.; Tran, A.T.; et al. Humoral and cellular immune responses against SARS-CoV-2 variants and human coronaviruses after single BNT162b2 vaccination. medrXiv 2021. [CrossRef]

78. Strengert, M.; Becker, M.; Ramos, G.M.; Dulovic, A.; Gruber, J.; Juengling, J.; Lürken, K.; Beigel, A.; Wrenger, E.; Lonnemann, G.; et al. Cellular and humoral immunogenicity of a SARS-CoV-2 mRNA vaccine in patients on hemodialysis. medrXiv 2021. [CrossRef]

79. Abu-Raddad, L.J.; Chemaitelly, H.; Butt, A.A. Effectiveness of the BNT162b2 Covid-19 Vaccine against the B.1.1.7 and B.1.351 Variants. N. Engl. J. Med. 2021. [CrossRef]

80. Xie, X.; Zou, J.; Fontes-Garfias, C.R.; Xia, H.; Swanson, K.A.; Cutler, M.; Cooper, D.; Menachery, V.D.; Weaver, S.D.; Dormitzer, P.R.; et al. Neutralization of N501Y mutant SARS-CoV-2 by BNT162b2 vaccine-elicited sera. biorXiv 2021. [CrossRef]

81. Collier, D.; Meng, B.; Ferreira, I.; Datir, R.; Temperton, N.J.; Elmer, A.; Graves, B.; Kingston, N.; McCoy, L.; Smith, K.; et al. Impact of SARS-CoV-2 B.1.1.7 Spike variant on neutralisation potency of sera from individuals vaccinated with Pfizer vaccine BNT162b2. medrXiv 2021. [CrossRef]

82. Muik, A.; Wallisch, A.-K.; Saenger, B.; Swanson, K.A.; Muehl, J.; Chen, W.; Cai, H.; Sarkar, R.; Tuereci, O.; Dormitzer, P.R.; et al. Neutralization of SARS-CoV-2 lineage B.1.1.7 pseudovirus by BNT162b2 vaccine-elicited human sera. Science 2021, 371, 1152-1153. [CrossRef] 
83. Lumley, S.F.; Rodger, G.; Constantinides, B.; Sanderson, N.; Chau, K.K.; Street, T.L.; O’Donnell, D.; Howarth, A.; Hatch, S.B.; Marsden, B.D.; et al. An observational cohort study on the incidence of SARS-CoV-2 infection and B.1.1.7 variant infection in healthcare workers by antibody and vaccination status. medrXiv 2021. [CrossRef]

84. Lopez Bernal, J.; Andrews, N.; Gower, C.; Gallagher, E.; Simmons, R.; Thelwall, S.; Tessier, E.; Groves, N.; Dabrera, G.; Myers, R.; et al. Effectiveness of COVID-19 vaccines against the B.1.617.2 variant. medRxiv 2021. [CrossRef]

85. Dejnirattisai, W.; Zhou, D.; Supasa, P.; Liu, C.; Mentzer, A.J.; Ginn, H.; Zhao, Y.; Duyvesteyn, H.M.E.; Tuekprakhon, A.; Nutalai, R.; et al. Antibody evasion by the Brazilian P.1 strain of SARS-CoV-2. medrXiv 2021. [CrossRef]

86. Ferreira, I.; Datir, R.; Papa, G.; Kemp, S.; Meng, B.; Rakshit, P.; Singh, S.; Pandey, R.; Ponnusamy, K.; Radhakrishnan, V.S.; et al. SARS-CoV-2 B.1.617 emergence and sensitivity to vaccine-elicited antibodies. medrXiv 2021. [CrossRef]

87. Edara, V.-V.; Lai, L.; Sahoo, M.; Floyd, K.; Sibai, M.; Solis, D.; Flowers, M.W.; Hussaini, L.; Ciric, C.R.; Bechnack, S.; et al. Infection and vaccine-induced neutralizing antibody responses to the SARS-CoV-2 B.1.617.1 variant. biorXiv 2021. [CrossRef]

88. Shi, P.-Y.; Liu, J.; Liu, Y.; Xia, H.; Zou, J.; Weaver, S.; Swanson, K.; Cai, H.; Cutler, M.; Cooper, D.; et al. Neutralization of SARS-CoV-2 variants B.1.617.1 and B.1.525 by BNT162b2-elicited sera. Res. Sq. 2021. [CrossRef]

89. Wu, K.; Werner, A.P.; Moliva, J.I.; Koch, M.; Choi, A.; Narayanan, E.; Stewart-Jones, G.B.E.; Colpitts, T.; Bennett, H.; BoyogluBarnum, S.; et al. Serum Neutralizing Activity Elicited by mRNA-1273 Vaccine. N. Engl. J. Med. 2021, 384, 1468-1470. [CrossRef]

90. Corbett, K.S.; Werner, A.; O'Connell, S.; Gagne, M.; Lai, L.; Moliva, J.I.; Flynn, B.; Choi, A.; Koch, M.; Foulds, K.E.; et al. Evaluation of mRNA-1273 against SARS-CoV-2 B.1.351 Infection in Nonhuman Primates. biorXiv 2021. [CrossRef]

91. Edara, V.V.; Hudson, W.H.; Xie, X.; Ahmed, R.; Suthar, M.S. Neutralizing Antibodies Against SARS-CoV-2 Variants After Infection and Vaccination. JAMA 2021, 325, 1896-1898. [CrossRef]

92. Shen, X.; Tang, H.; McDana, C.; Wagh, K.; Fischer, W.; Theiler, J.; Yoon, H.; Li, D.; Haynes, B.F.; Sanders, K.O.; et al. SARS-CoV-2 variant B.1.1.7 is susceptible to neutralizing antibodies elicited by ancestral Spike vaccines. Cell Host Microbe 2021, 29, 529-539. [CrossRef]

93. Madhi, S.A.; Baillie, V.; Cutland, C.L.; Voysey, M.; Koen, A.L.; Fairlie, L.; Padayachee, S.D.; Dheda, K.; Barnabas, S.L.; Bhorat, Q.E.; et al. Efficacy of the ChAdOx1 nCoV-19 Covid-19 Vaccine against the B.1.351 Variant. N. Engl. J. Med. 2021. [CrossRef]

94. Fischer, R.; van Doremalen, N.; Adney, D.; Yinda, C.K.; Port, J.R.; Holbrook, M.G.; Schulz, J.E.; Williamson, B.N.; Thomas, T.; Barbian, K.; et al. ChAdOx1 nCoV-19 (AZD1222) protects against SARS-CoV-2 B.1.351 and B.1.1.7. biorXiv 2021. [CrossRef]

95. Yadav, P.; Sapkal, G.N.; Abraham, P.; Deshpande, G.; Nyayanit, D.A.; Patil, D.Y.; Gupta, N.; Sahay, R.R.; Shete, A.M.; Kumar, S.; et al. Neutralization potential of Covishield vaccinated individuals against B.1.617.1. Clin. Infect. Dis. 2021, ciab483. [CrossRef]

96. Ikegame, S.; Siddiquey, M.N.A.; Hung, C.-T.; Haas, G.; Brambilla, L.; Oguntunyo, K.Y.; Kowdle, S.; Vilardo, A.E.; Edelstein, A.; Perandones, C.; et al. Qualitatively distinct modes of Sputnik V vaccine-neutralization escape by SARS-CoV-2 Spike variants. medrXiv 2021. [CrossRef]

97. Shinde, V.; Bhikha, S.; Hossain, Z.; Archary, M.; Bhorat, Q.; Fairlie, L.; Lalloo, U.; Masilela, M.S.L.; Moodley, D.; Hanley, S.; et al. Efficacy of NVX-CoV2373 Covid-19 Vaccine against the B.1.351 Variant. N. Engl. J. Med. 2021, 384, 1899-1909. [CrossRef]

98. Sapkal, G.N.; Yadav, P.; Ella, R.; Deshpande, G.R.; Sahay, R.R.; Gupta, N.; Mohan, V.K.; Abraham, P.; Panda, S.; Bhargava, B. Neutralization of UK-variant VUI-202012/01 with COVAXIN vaccinated human serum. medrXiv 2021. [CrossRef]

99. Chen, Y.; Shen, H.; Huang, R.; Tong, X.; Wu, C. Serum neutralising activity against SARS-CoV-2 variants elicited by CoronaVac. Lancet Infect. Dis. 2021. [CrossRef]

100. Moore, P.; Moyo, T.; Hermanus, T.; Kgagudi, P.; Ayres, F.; Makhado, Z.; Sadoff, J.; Le Gars, M.; van Roey, G.; Crowther, C.; et al. Neutralizing antibodies elicited by the Ad26.COV2.S COVID-19 vaccine show reduced activity against 501Y.V2 (B.1.351), despite protection against severe disease by this variant. biorXiv 2021. [CrossRef] 